\title{
LA EXPOSICIÓN ORAL: VALORAR Y SER VALORADO
}

Oral presentations: Assessing and being assesed

A apresentação oral: Availar e ser avaliado

Camino Fierro (1)

Vicente Martínez (2)

(1) Departamento de Biología Celular, Fisiología e Inmunología. Universidad Autónoma de Barcelona. Teléfono: 93581 1664. Correo electrónico:

\section{mariacamino.fierro@uab.es}

(2) Departamento de Biología Celular, Fisiología e Inmunología. Instituto de Neurociencias. Universidad Autónoma de Barcelona. Teléfono: 93581 3834. Correo electrónico: vicente.martinez@uab.es

\section{Resumen}

En esta experiencia de evaluación de exposiciones orales, se intenta dar un mayor peso a la figura de los estudiantes que escuchan las presentaciones, trabajando de manera participativa y bidireccional la labor de evaluación. La actividad de exposición oral, no solo consta de la exposición en público, sino que se completa con las siguientes fases, la creación previa en común de la plantilla de evaluación, donde se consensuan los criterios de evaluación; una dinámica de puntuación de las plantillas por las que obtendrán la nota de evaluación y una fase final de lectura de la evaluación de sus compañeros, generando así una actividad de evaluación participativa. Logramos así no solo hacer una actividad de exposición oral, sino también una participación más activa en las exposiciones de los compañeros, la adquisición de habilidades para generar una opinión y obtener una visión más global de su propio trabajo de exposición y no solo desde el punto de vista del profesor.

Palabras clave: Exposición oral; evaluación participativa; valoración

\begin{abstract}
In this experience of evaluation of oral presentations, we try to emphasize the role of the listeners, working in a participatory environment and in making the process of


evaluation bidirectional. The oral exposition activity, consists not only of a public presentation, but also in the preliminary group creation of an evaluation template, where the points to be assessed are agreed upon and after which their own oral presentations will be evaluated; the scoring of the templates for which they will obtain the evaluation mark, and final phase of reading their peer's evaluation of their own presentation. With this strategy, we perform an oral presentation activity, promoting, at the same time, a more active participation of the students in the presentations of their classmates, leading to the development of skills related to opinion generation and independent selfevaluation.

Keywords: Oral presentation; participatory evaluation; assessment

\section{Resumo}

Nesta experiência de avaliação de apresentações orais, tenta-se dar um maior peso à de alunos que ouvir as apresentações, trabalhando de forma participativa e bidirecional o trabalho da avaliação. A atividade de exposição oral não só consta da exposição oral em público, mas completa-se com a criação prévia de um modelo de avaliação, em que se estabelecem os pontos a valorizar e quais são avaliadas as suas próprias apresentações orais; adinâmica de pontuação do modelo pelo que obtem a nota de avaliação final de uma fase final de leutira da avaliação dos seus companheiros. Conseguimos desta forma fazer não apenas unma avaliação de exposição oral, mas também uma participação mais ativa nas exposições dos companheiros, a aquisição de habilidades para gerar uma opinião e obter uma visão mais global do seu próprio trabalho de exposição e não so desde o ponto de vista do professor.

Palavras-chave: Apresentação oral; avaliação participativa; avaliação

\section{Introducción}

La comunicación oral cumple una función comunicativa, en cuanto sirve como instrumento para enseñar, evaluar y hacer público el conocimiento (Peña Borrero, 2008).

La exposición oral, además de ser una competencia instrumental importante dentro del Espacio Europeo de Educación Superior (EEES), es fundamental en el 
desarrollo de la carrera investigadora como mecanismo para dar a conocer a la comunidad científica su trabajo. La exposición oral es siempre un reto difícil de asumir, donde no solo debe trabajar el comunicador, sino también los receptores de la comunicación oral evaluando el trabajo.

La evaluación formativa hace referencia a sistemas de evaluación cuya principal finalidad es mejorar el aprendizaje del alumnado y el funcionamiento del proceso de enseñanza y aprendizaje (López-Pastor, 2006). Además, la evaluación participativa, "busca la incorporación activa y consciente de los miembros de la organización en el proceso evaluativo” (Rivas \& Donovan, 2001, p. 26).

En este estudio se presentan los resultados de una actividad que intenta incentivar estas dos facetas en la educación, la presentación oral y el proceso de evaluación participativa.

\section{Contextualización}

Esta experiencia se ha llevado a cabo en las sesiones de seminarios de la asignatura de Fisiología Animal del segundo curso del Grado en Biotecnología de la Universidad Autónoma de Barcelona a lo largo de cuatro cursos, desde 2013/14 hasta el actual 2016/17.

La exposición oral es una de las actividades programadas para trabajar las habilidades de comunicación oral en público. Para esta actividad la clase $(70 \pm 3$ alumnos/año) se divide en dos grupos (de $30 \pm 5$ estudiantes) y cada grupo en subgrupos de 4-5 estudiantes (8 subgrupos por grupo). Cada grupo dispone de 4 sesiones (2 exposiciones por sesión de $50 \mathrm{~min}$ ).

\section{Diseño y desarrollo}

Las exposiciones orales son puntuadas por el profesor y suponen un $40 \%$ de la nota de seminarios. Aprovechando esta actividad, se desarrolla el trabajo de evaluación participativa. Por la labor de evaluación, los alumnos obtienen una calificación que supone un $10 \%$ de la nota final de seminarios.

Antes del comienzo de las exposiciones orales, se realiza una sesión para explicar y establecer cómo será la actividad de evaluación. Se presenta al alumnado la plantilla de evaluación que se utilizará, tanto por el propio profesor como por los 
estudiantes, y se consensuan los criterios de evaluación (Fig 1.). La plantilla incluye una nota final y un comentario crítico sobre la exposición oral. Este modelo se rellena para cada exposición oral y una por subgrupo. Concluidas las exposiciones, en una sesión final, todos los grupos reciben, de forma anónima, las calificaciones y los comentarios de sus compañeros.

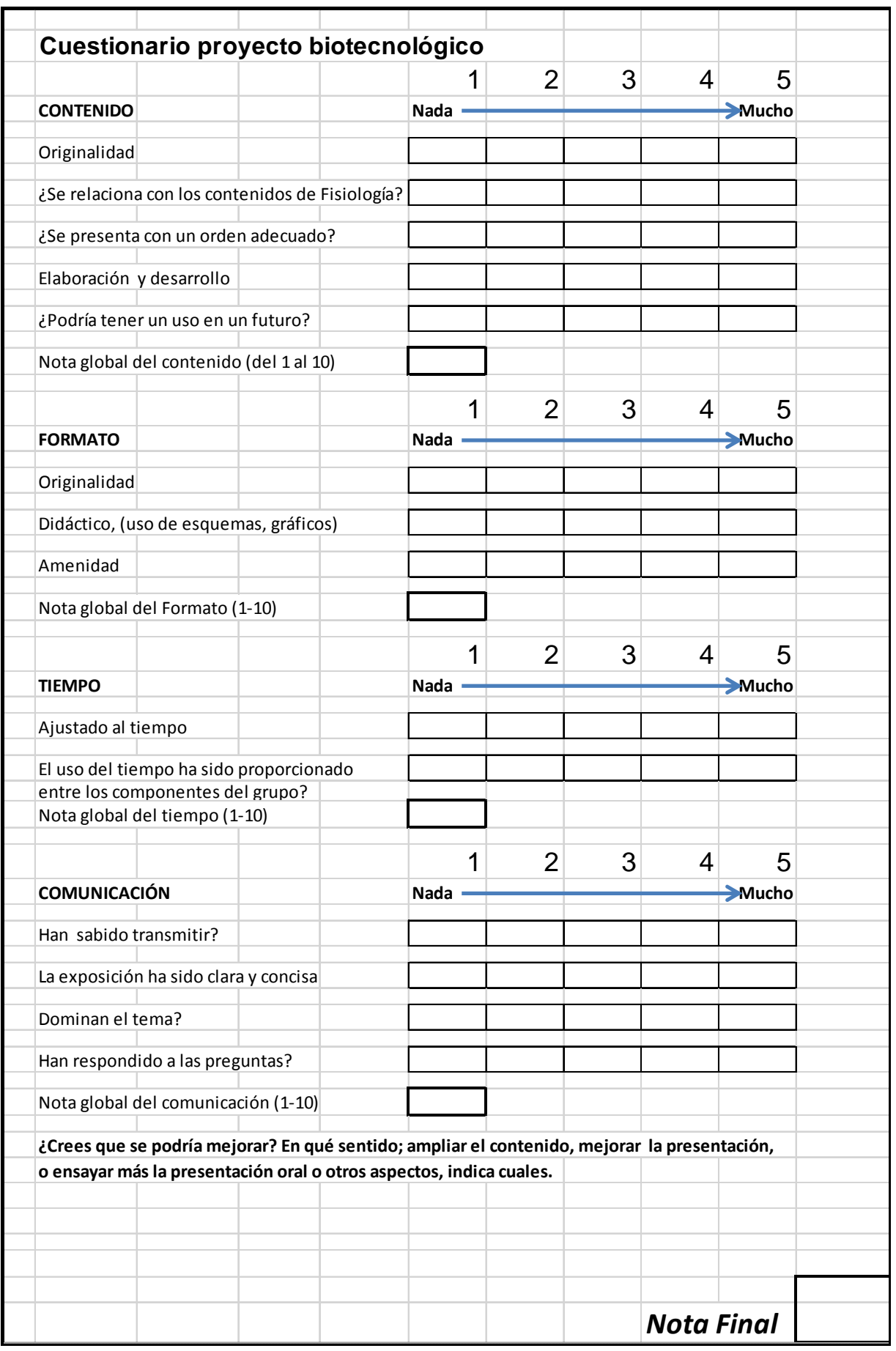

\section{Figura 1.}

Plantilla de evaluación 
En paralelo, el profesor otorga a cada estudiante una calificación por su labor de evaluación. Esta se genera de la diferencia entre la nota que el profesor da al grupo que expone y la nota que da los estudiantes a ese mismo grupo. Así, si ambas notas coinciden, la diferencia será 0 , el grupo evaluador obtiene la máxima calificación, 10. Por tanto, a medida que las diferencias entre la evaluación del profesor y el grupo se incrementan, la nota que se obtiene se reduce. Con lo cual se impone un criterio de equidad en la evaluación, que sobre todo sirve para evitar una valoración desmedida de las exposiciones por parte de los compañeros

\section{Evaluación}

La asistencia a las sesiones de exposición oral, aun no siendo obligatorias, fue de un 95\% (solo se exige un $50 \%$ de los componentes de cada subgrupo para rellenar y entregar la plantilla de evaluación). La primera sesión siempre resulta más compleja, es el primer momento en el que se enfrentan a la valoración sobre la exposición oral de sus propios compañeros. Es con el progreso de las sesiones orales cuando el sentido crítico va incrementándose, lo que hace que su puntuación y la del profesor se vayan aproximando (Fig. 2), no siendo nunca esta diferencia mayor de dos puntos, y que sus comentarios sean más enriquecedores.

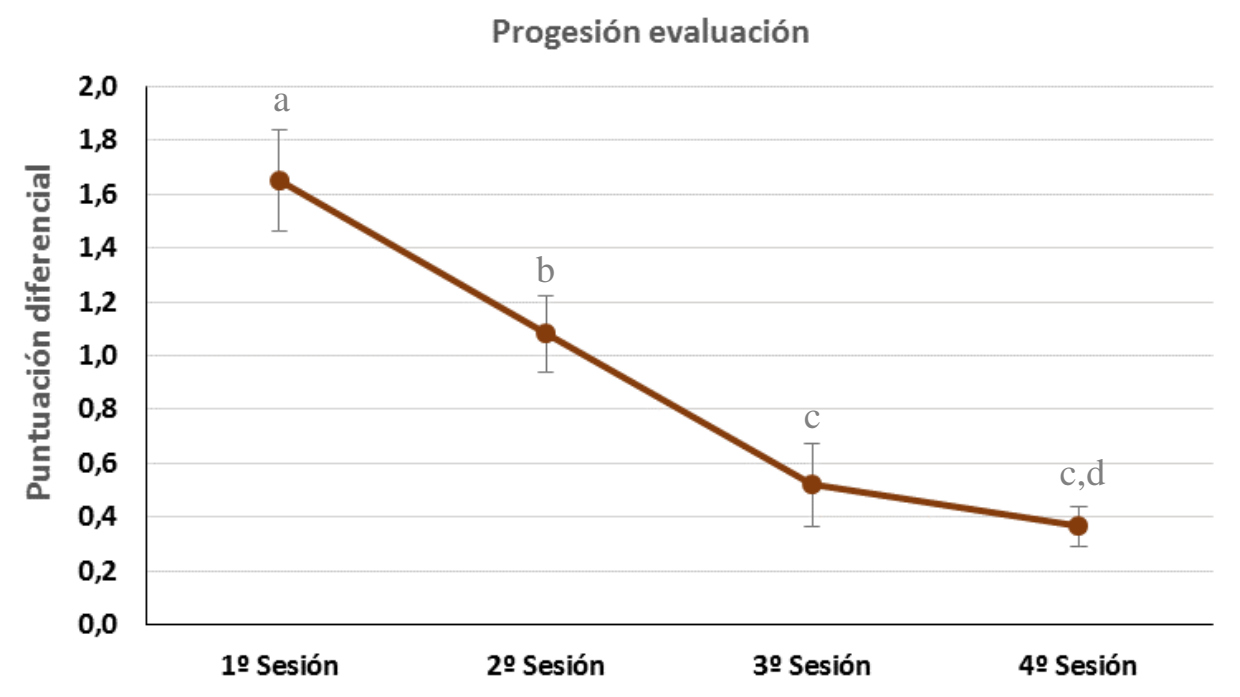

Figura 2.

Progresión de la evaluación durante las sesiones. Cada valor representa la media y su desviación estándar de las diferencias obtenidas entre la puntación del profesor y los estudiantes. Las letras marcan las diferencias significativas $(P<0,01)$ entre las distintas sesiones mediante análisis de varianza. Los puntos que comparten letras no presentan diferencias significativas $N=16$ 
Una de las mayores preocupaciones de los alumnos es que su percepción y la del profesor difieran en gran medida siendo algo más acentuada dicha diferencia en las primeras sesiones (Fig.2). Sin embargo con el trascurso de las sesiones la diferencia se acorta y la nota final resultante de la evaluación participativa es siempre de \pm 9.3 sobre 10 (Tabla 1).

Tabla 1.

Calificación final por la evaluación

\begin{tabular}{llc}
\hline Curso académico & Número de grupos & $\begin{array}{c}\text { Calificación } \\
\text { (media } \pm \text { SEM) }\end{array}$ \\
\hline $2013-2014$ & 16 & $9.21 \pm 0.28$ \\
$2014-2015$ & 16 & $9.26 \pm 0.23$ \\
$2015-2016$ & 16 & $9.44 \pm 0.20$ \\
$2016-2017$ & 16 & $9.56 \pm 0.21$ \\
\hline
\end{tabular}

Los valores corresponden a la media de todos los sub-grupos

\section{Conclusiones}

Una de las primeras consecuencias que se detectaron fue la alta asistencia de los alumnos a las exposiciones orales, saber que sus compañeros les van a evaluar parece favorecer la participación en el proceso de valorar y ser valorado.

Otro hecho que se evidenció es como el conocer con antelación los criterios de evaluación, ayuda a su mejora. El poder valorarlo en los demás ayuda a la autocrítica. No obstante en esta última cuestión los primeros grupos que exponen se ven algo menos favorecidos al no contar con un referente previo.

Las puntaciones obtenidas por la actividad de evaluación, han servido para ayudar a incrementar la calificación final de seminarios.

Uno de los objetivos principales es implicar a los alumnos en el proceso de evaluación participativa. Con este proceso consiguen conocer la opinión de un mayor público y no únicamente la del profesor en una última sesión de seminarios donde pueden ver los cuestionarios (anónimos) que han rellenado sus compañeros respecto a su exposición oral, y así poder analizar los puntos donde ellos mismos deben mejorar para futuras exposiciones orales.

Con todo ello se logra que una actividad como son las exposiciones orales, desarrolle de manera efectiva competencias tanto específicas de la materia como transversales. 


\section{Referencias}

Peña Borrero, L. B. (2008). La competencia oral y escrita en la educación superior. Recuperado de http://www.mineducacion.gov.co/1621/articles189357_archivo_pdf_comunicacion.pdf

López Pastor, V.M. (2006). La Evaluación en Educación Física. Revisión de los modelos tradicionales y planteamiento de una alternativa: la evaluación formativa y compartida. Buenos Aires: Miñó y Dávila.

Rivas, M. T., \& Donovan P. (2001). La evaluación participativa (2a ed.). Quito, Ecuador: Avia-Yala. 